\title{
Factores predictores de éxito en litotripsia extracorpórea por ondas de choque (LEOCH)
}

Berber-Deseusa A, ${ }^{1}$ Maldonado-Ávila $M,{ }^{2}$ Garduño-Arteaga $M L,{ }^{3}$ JaspersenGastelum J, ${ }^{4}$ Virgen-Gutiérrez F, ${ }^{4}$ Rodríguez-Nava $\mathrm{P}^{5}$

\section{Resumen}

ANTECEDENTES: la litiasis urinaria constituye una de las enfermedades urológicas de mayor atención médica en México. La tomografía computada es el estudio de referencia para establecer el diagnóstico de la enfermedad, pues permite conocer la densidad, carga litiásica, tamaño, localización y distancia piel-cálculo.

OBJETIVO: identificar si el tamaño, localización, densidad del cálculo urinario, distancia piel-calculo e IMC representan factores predictores de éxito de la litotripsia extracorpórea por ondas de choque (LEOCH), además de estimar la eficacia del equipo Litotriptor Piezolith 3000 plus (Richard Wolf).

MATERIALES Y MÉTODOS: estudio transversal, analítico, efectuado en pacientes con diagnóstico de litiasis urinaria, atendidos en el servicio de Urología del Hospital General de México, de diciembre de 2013 a junio de 2015, para recibir litotripsia extracorpórea por ondas de choque. Se consideró "éxito" a la eliminación completa del cálculo y "fracaso" a la identificación de cualquier fragmento litiásico residual. Se utilizó el modelo de regresión logística binaria para establecer la asociación entre el éxito de la LEOCH y las variables de estudio.

RESULTADOS: de 607 LEOCH efectuadas, solo 60 pacientes cumplieron con los criterios de inclusión: 30 tuvieron fragmentación y eliminación completa del cálculo y 30 litiasis residual. El modelo de regresión logística binaria mostró que el sexo, IMC, localización y promedio del cálculo, distancia piel-cálculo y número de golpes, no se consideran factores predictores de éxito para LEOCH versus las unidades Hounsfield, que son estadísticamente significativas del éxito en LEOCH ( $p=0.01$; RM de 6; IC 95\% 1.4-26.2).

CONCLUSIONES: la densidad del cálculo $<1000$ UH representa un factor predictor de éxito para LEOCH. La distancia piel-cálculo, IMC, tamaño y localización del cálculo no se relacionan con el éxito de la LEOCH. La eficacia global del litotriptor PiezoLith 3000 plus (Richard Wolf) es de $50 \%$.

PALABRAS CLAVE: litiasis urinaria, litotripsia extracorpórea por ondas de choque, factores predictores de éxito.

\footnotetext{
${ }^{1}$ Adscrito al servicio de Urología, Hospital Regional de Tlalnepantla (ISSEMYM).

${ }^{2}$ Urólogo, profesor adjunto del posgrado de Urología (UNAM), coordinador de investigación de urología. ${ }^{3}$ Jefe del servicio de Urología.

${ }^{4}$ Adscrito a la sala de litiasis urinaria.

${ }^{5}$ Jefa del Departamento de Tomografía Computada.
}

Hospital General de México Dr. Eduardo Liceaga, Ciudad de México.

Recibido: mayo 2017

Aceptado: junio 2017

Correspondencia

Dr. Anuar Berber Deseusa

dranuarberber@gmail.com

Este artículo debe citarse como

Berber-Deseusa A, Maldonado-Ávila M, GarduñoArteaga ML, y col. Factores predictores de éxito en litotripsia extracorpórea por ondas de choque (LEOCH). Rev Mex Urol. 2017 jul-agos;77(4):251-257. DOI: https://doi.org/10.24245/revmexurol.v77i4.1441 


\section{Predictive success factors in extracorporeal shock wave lithotripsy (ESWL)}

Berber-Deseusa A, ${ }^{1}$ Maldonado-Ávila $\mathrm{M},{ }^{2}$ Garduño-Arteaga $\mathrm{ML},{ }^{3}$ JaspersenGastelum J, ${ }^{4}$ Virgen-Gutiérrez $F^{4}{ }^{4}$ Rodríguez-Nava $P^{5}$

\begin{abstract}
BACKGROUND: Urinary lithiasis is one of the most widely treated urologic diseases in Mexico. Computed tomography is the criterion standard in its diagnosis, identifying density, stone burden, size, location, and skin-to-stone distance.

OBJECTIVE: The aims of the present study were to determine whether size, location, density, and skin-to-stone distance, as well as the patient's body mass index, were predictive success factors following a single extracorporeal shock wave lithotripsy session and to establish the efficacy of the Richard Wolf Piezolith 3000 lithotripter.
\end{abstract}

MATERIALS AND METHODS: A cross-sectional, analytic study was conducted on patients diagnosed with urinary lithiasis that underwent extracorporeal shock wave lithotripsy, seen at the Urology Service of the Hospital General de México within the time frame of December 2013 and June 2015. "Success" was considered when there was complete stone elimination and "failure" was the presence of any residual stone fragments. A binary logistic regression model was employed to establish the association between extracorporeal shock wave lithotripsy success and the study variables.

RESULTS: Of the 607 extracorporeal shock wave lithotripsies performed, only 60 patients met the inclusion criteria: 30 had fragmentation and complete elimination of the stone and 30 had residual lithiasis. The binary logistic regression model showed that sex, body mass index, stone location and average size, mean skin-to-stone distance, and number of shock wave hits could not be considered success factors for extracorporeal shock wave lithotripsy. In contrast, Hounsfield units were statistically significant in relation to extracorporeal shock wave lithotripsy success $(\mathrm{p}=0.01)(\mathrm{OR}$ 6; 95\% Cl: 1.4-26.2).

CONCLUSIONS: Stone density $<1000 \mathrm{HU}$ was a predictive success factor for extracorporeal shock wave lithotripsy. Skin-to-stone distance, body mass index, stone size, and stone location were not related to extracorporeal shock wave lithotripsy success. The overall efficacy of the Richard Wolf PiezoLith 3000 lithotripter was 50\%.

KEYWORDS: Urinary lithiasis; Extracorporeal shock wave lithotripsy; Predictive factors for success

\footnotetext{
${ }^{1}$ Adscrito al servicio de Urología, Hospital Regional de Tlalnepantla (ISSEMYM). 2 Urólogo, profesor adjunto del posgrado de Urología (UNAM), coordinador de investigación de urología.

${ }^{3}$ Jefe del servicio de Urología.

${ }^{4}$ Adscrito a la sala de litiasis urinaria.

${ }^{5}$ Jefa del Departamento de Tomografía Computada.
}

Hospital General de México Dr. Eduardo Liceaga, Ciudad de México.

\section{Correspondence}

Dr. Anuar Berber Deseusa dranuarberber@gmail.com 


\section{ANTECEDENTES}

La litiasis urinaria es la tercera enfermedad con mayor demanda de atención médica, después de las infecciones de vías urinarias y enfermedad prostática. En algunas zonas de México la prevalencia es mayor a la reportada en la bibliografía mundial. ${ }^{1}$

La tomografía de abdomen y pelvis es el estudio de referencia para establecer el diagnóstico de litiasis urinaria, pues ofrece alta sensibilidad y especificidad (95 y $98 \%$, respectivamente). El estudio permite determinar la localización, número, tamaño y forma de los cálculos urinarios, además de conocer el valor de atenuación (expresado en unidades Hounsfield), con la finalidad de proveer información pronóstica de la fragilidad del cálculo y el éxito de la litotripsia extracorpórea por ondas de choque (LEOCH) ${ }^{2-6}$

Existen diversas opciones de tratamiento de la litiasis urinaria; sin embargo, la LEOCH es el procedimiento de primera línea para la litiasis renal o ureteral. ${ }^{7-8}$ La decisión del tratamiento se establece con base en el tamaño, número y localización de los cálculos en la vía urinaria. No obstante, el tratamiento inicial con LEOCH ha demostrado numerosas fallas terapéuticas, ya sea por la falta de fragmentación completa o incapacidad para eliminar todos los fragmentos listiásicos. ${ }^{3}$

La determinación de la densidad, carga litiásica, tamaño, localización y distancia piel-cálculo mediante tomografía preoperatoria permite predecir el éxito de la LEOCH. ${ }^{2-4}$ Por lo tanto, puede estimar qué o cuántos pacientes son ideales para recibir el tratamiento y obtener mejores resultados.

El objetivo primario de este estudio fue identificar si el tamaño, localización, densidad del cálculo urinario, distancia piel-cálculo e IMC constituyen factores predictores de éxito después de una sesión única de litotripsia extracorpórea por ondas de choque (LEOCH), y el objetivo secundario: establecer la eficacia del equipo Litotriptor Piezolith 3000 plus (Richard Wolf).

\section{MATERIALES Y MÉTODOS}

Estudio transversal y analítico efectuado en pacientes con diagnóstico de litiasis urinaria, atendidos en el servicio de Urología del Hospital General de México Dr. Eduardo Liceaga, de diciembre de 2013 a junio de 2015. El diagnóstico de litiasis renal o ureteral en el tercio superior fue confirmado con radiografía de abdomen y tomografía simple de abdomen y pelvis. Los pacientes con litiasis múltiple, litiasis radiolúcida, cálculos coraliformes o mayores de $20 \mathrm{~mm}$ fueron excluidos del estudio.

Los pacientes seleccionados fueron intervenidos con la técnica de litotripsia extracorpórea por ondas de choque (LEOCH), con el equipo litotriptor PiezoLith 3000 plus (Richard Wolf), a quienes se aplicaron ondas de choque de 200 a 4000 golpes. Durante la LEOCH, los pacientes recibieron analgesia con diclofenaco intramuscular o bloqueo del duodécimo nervio intercostal con lidocaína simple al $2 \%$ y analgésicos intravenosos (tramadol o ketorolaco). Se definió "éxito" de la técnica a la eliminación completa del cálculo, comprobado con radiografía de abdomen después de dos semanas de haber realizado litotripsia. Se definió "fracaso" a la coexistencia de litiasis residual, sin importar su tamaño.

Las variables preoperatorias analizadas fueron: sexo, IMC, tamaño máximo del cálculo, volumen litiásico, distancia piel-cálculo, densidad y localización.

\section{Análisis estadístico}

Los valores se expresaron como medias $( \pm$ ) y desviación estándar (DE). Se utilizó la prueba t de 
Student para la comparación de medias independientes y el modelo de regresión logística binaria para establecer la asociación entre el éxito de la LEOCH y las variables de estudio. Para el análisis de los datos se utilizó el programa estadístico IBM SPSS Statistics 20 para Mac (SPSS, Chicago, IL). Se consideró estadísticamente significativo el valor de $\mathrm{p}<0.05$.

\section{RESULTADOS}

De diciembre de 2013 a junio de 2015 se realizaron $607 \mathrm{LEOCH}$, de las que solo 60 pacientes cumplieron con los criterios de inclusión: 42 mujeres y 18 hombres. La localización más frecuente de la litiasis fue en la pelvis renal (27 casos), seguida del tercio superior del uréter (15 casos), cáliz inferior (15 casos), cáliz medio (2 casos) y cáliz superior (1 caso).

Aunque 47 (78.3\%) pacientes tuvieron derivaciones urinarias (42 con colocación de catéter JJ y 5 nefrostomía), no representó un factor implicado en el resultado final de la $\mathrm{LEOCH}$.

De acuerdo con la definición de "éxito" o "fraca-

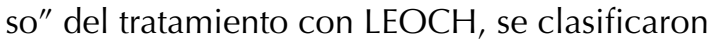
las variables para formar dos grupos: 1) pacientes libres de litiasis posterior a la LEOCH (30 casos) y 2) pacientes con litiasis residual posterior a la LEOCH (30 casos).

No se encontraron diferencias estadísticamente significativas en cuanto a edad, promedio y volumen litiásico, distancia piel-cálculo, densidad del cálculo, intensidad de las ondas de choque e IMC. Aunque tampoco se registraron diferencias estadísticamente significativas en la longitud máxima del cálculo ( $p=0.057)$, se observó una tendencia mayor en el grupo con litiasis residual (Cuadro 1).

Respecto del número de ondas de choque (golpes) existieron diferencias estadísticamente
Cuadro 1. Características demográficas

\begin{tabular}{|c|c|c|c|}
\hline $\begin{array}{l}\text { Variables } \\
(n=60)\end{array}$ & $\begin{array}{c}\text { Sin litiasis } \\
\text { residual } \\
(n=30) \\
\text { promedio } \pm \text { DE }\end{array}$ & $\begin{array}{c}\text { Con litiasis } \\
\text { residual } \\
(\mathbf{n}=30) \\
\text { promedio } \pm \mathrm{DE}\end{array}$ & $\mathbf{p}$ \\
\hline Edad (años) & $44.4 \pm 11.9$ & $49.0 \pm 12.9$ & 0.156 \\
\hline $\begin{array}{l}\text { Longitud máxima } \\
\text { del lito }(\mathrm{mm})\end{array}$ & $12.8 \pm 3.6$ & $14.6 \pm 3.4$ & 0.057 \\
\hline $\begin{array}{l}\text { Promedio del } \\
\text { cálculo }(\mathrm{mm})\end{array}$ & $11.1 \pm 3.1$ & $12.1 \pm 2.7$ & 0.160 \\
\hline $\begin{array}{l}\text { Volumen litiásico } \\
\left(\mathrm{mm}^{3}\right)\end{array}$ & $871.7 \pm 837.4$ & $1028.3 \pm 708.7$ & 0.437 \\
\hline $\begin{array}{l}\text { Distancia piel- } \\
\text { cálculo (mm) }\end{array}$ & $102 \pm 22.2$ & $102.8 \pm 24$ & 0.912 \\
\hline $\begin{array}{l}\text { Densidad del } \\
\text { cálculo }(U H)\end{array}$ & $917 \pm 301$ & $1058 \pm 285$ & 0.068 \\
\hline IMC $\left(\mathrm{kg} / \mathrm{m}^{2}\right)$ & $28.6 \pm 5.02$ & $29.8 \pm 4.4$ & 0.326 \\
\hline $\begin{array}{l}\text { Intensidad } \\
(\mathrm{kv})\end{array}$ & $15.6 \pm 2.0$ & $15.6 \pm 1.88$ & 0.40 \\
\hline
\end{tabular}

*Nivel de significancia $<0.05$

significativas $(p=0.013)$ entre el grupo libre de litiasis $(2631 \pm 1057)$ vs litiasis residual $(3202$ \pm 632 ). Figura 1

El modelo de regresión logística binaria (Cuadro 2) mostró que el sexo, IMC, localización

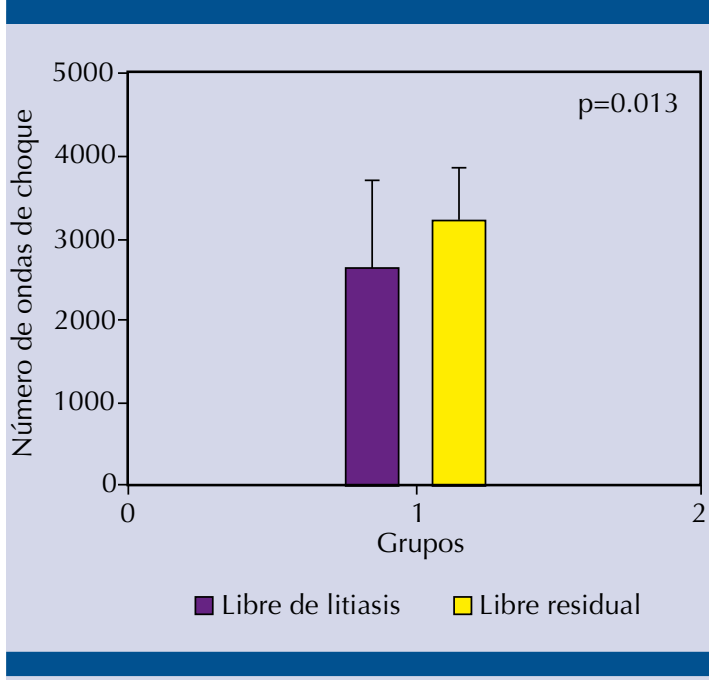

Figura 1. Promedio de ondas de choque por grupo. 
Cuadro 2. Análisis multivariado

\begin{tabular}{|l|c|c|c|}
\hline Variables & OR & IC 95\% & p \\
\hline Sexo & 0.63 & $0.15-2.50$ & 0.51 \\
\hline IMC & 0.75 & $0.51-1.10$ & 0.14 \\
\hline Localización del cálculo & 0.45 & $0.18-1.15$ & 0.09 \\
\hline Promedio del lito & 0.73 & $0.13-3.90$ & 0.72 \\
\hline Distancia piel-cálculo & 1.57 & $0.40-6.18$ & 0.51 \\
\hline Unidades Hounsfield & 6 & $1.40-26.2$ & 0.01 \\
\hline Golpes/pulsos (onda) & 3.39 & $0.75-15.40$ & 0.11
\end{tabular}

*Nivel de significancia $<0.05$. Análisis de regresión logística binaria de las variables de estudio como factores predictores de éxito en LEOCH.

y promedio del cálculo, distancia piel-cálculo y número de ondas de choque (golpes) no se consideran factores predictores de éxito para la LEOCH, pero las unidades Hounsfield sí pueden considerarse un factor de éxito ( $p=0.01$ ) (RM 6, IC 95\% 1.4-26.2).

El $66.7 \%$ de los pacientes del grupo de litiasis residual tuvo cálculos con más de 1000 unidades Hounsfield (Cuadro 3, Figura 2).

\section{DISCUSIÓN}

Existen varias opciones de tratamiento para la litiasis urinaria. La LEOCH se considera la primera línea de tratamiento para litiasis renal o ureteral, ${ }^{3,7,8}$ pues constituye un procedimiento menos invasivo, comparado con otras modalidades como la ureterolitotripsia y nefrolitotomía percutánea, aunque con ciertas limitaciones

Cuadro 3. Pacientes con base en la densidad del cálculo por grupo de estudio

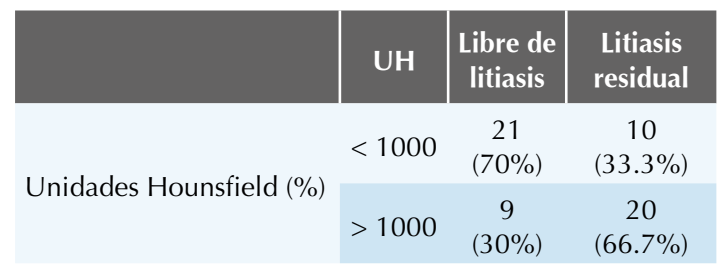

UH: unidades Hounsfield

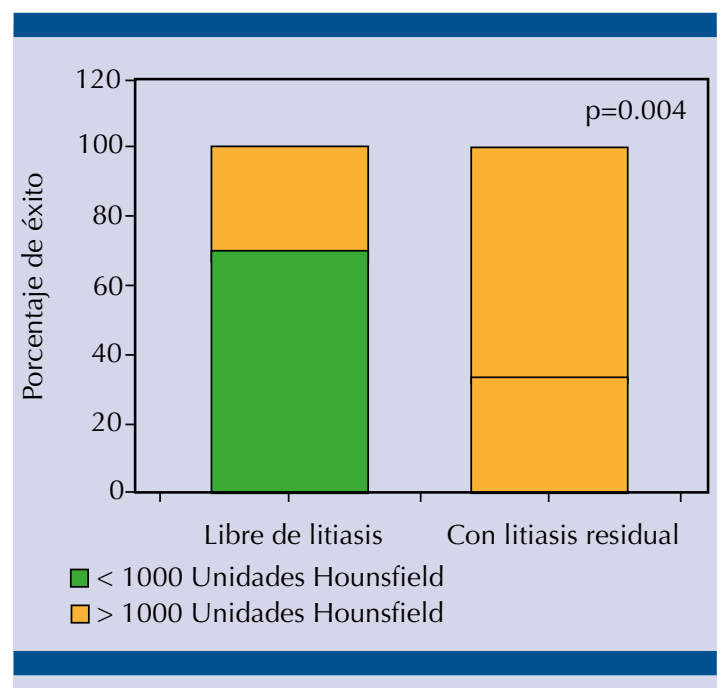

Figura 2. Distribución de pacientes por grupo y densidad del cálculo expresado en unidades Hounsfield.

en lo que a eficacia respecta. ${ }^{7}$ La decisión del tratamiento se establece con base en el tamaño, número y localización de los cálculos en la vía urinaria.

En este estudio se encontraron diferencias estadísticamente significativas en cuanto al número de golpes-pulsos u ondas de choque. Paradójicamente, los pacientes con litiasis residual recibieron mayor número de golpes, quizá debido a que los cálculos eran de mayor longitud.

También se observaron diferencias estadísticamente significativas en los pacientes con cálculos con unidades Hounsfield mayores de 1000, situación que coincide con lo reportado por Wiesenthal y sus coautores, quienes demostraron que la medición de la densidad de los cálculos (<900 UH) constituye un factor predictor de éxito en la LEOCH para cálculos renales y ureterales. ${ }^{3}$ En relación con el tamaño del cálculo, aunque no se encontraron diferencias estadísticamente significativas, se observó una tendencia de mayor dimensión en el grupo de litiasis residual. 
El estudio de Cakiroglu y su grupo señala que el tamaño del cálculo es un factor predictor de éxito de la LEOCH en el tratamiento de cálculos ureterales, con mayor falla en litos $>10 \mathrm{~mm}^{2}$

Kanao y sus colaboradores demostraron que el tamaño del cálculo, la localización y el número de litos son factores predictores en la tasa libre de litiasis. ${ }^{7}$ Con base en esto se considera que los pacientes con cálculos $>10 \mathrm{~mm}$ tienen mayor riesgo de fracaso al tratamiento con $\mathrm{LEOCH} .{ }^{9}$ Aunque esta situación no se confirmó en nuestro estudio, consideramos que al aumentar el tamaño de la muestra pueda hacerse evidente.

No encontramos diferencias estadísticamente significativas en la localización del cálculo, comparado con el informe de Weld y su grupo, quienes reportaron que los cálculos localizados en el cáliz inferior son de peor pronóstico. ${ }^{10}$ Tampoco encontramos correlación estadísticamente significativa en cuanto al IMC como factor predictivo de éxito en la $\mathrm{LEOCH}$, resultado que coincide con Perks y su equipo, quienes demostraron pequeñas variaciones en el IMC, que no afectaron el resultado de la técnica. ${ }^{11}$

La eficacia global del litotriptor PiezoLith 3000 plus (Richard Wolf) fue de $50 \%$; si bien algunos artículos reportan tasas libres de litiasis entre $68-80 \%$, ${ }^{12-14}$ otros (incluidos los últimos nomogramas para $\mathrm{LEOCH}$ ) reportan valores de $37-50 \%{ }^{5,11,15} \mathrm{El}$ estudio aquí realizado puede servir como base de las características especificas de nuestra población y generar factores predictores ajustados a éstas, para así limitar la utilización de esta técnica en pacientes con perfil más favorable y mejorar los resultados de la LEOCH, como lo sugieren Bengio y su grupo. ${ }^{16}$

Entre las fortalezas de nuestro estudio encontramos que las mediciones: distancia piel-cálculo, densidad del cálculo expresado en unidades Hounsfield (UH) y longitud del cálculo mediante tomografía simple de abdomen y pelvis son acce- sibles para realizarlas en la consulta de urología y sirvan para establecer el tratamiento de litiasis renal y ureteral como factores predictores de éxito en $\mathrm{LEOCH}$.

Una de las debilidades del estudio fue el tamaño pequeño de la muestra, comparada con estudios similares publicados a nivel mundial. Esto se debe a que un gran porcentaje de los pacientes que acuden a nuestro servicio para $\mathrm{LEOCH}$, les fue realizada la tomografía en los hospitales de referencia; por lo tanto, no contábamos con todas las variables para incluirlas en el protocolo. Sin embargo, este estudio puede servir de precedente para estudios futuros en nuestro hospital, ya que no se cuenta con reportes nacionales relacionados con factores predictores de éxito en pacientes intervenidos de LEOCH.

\section{CONCLUSIONES}

La densidad del cálculo $<1000$ UH es un factor predictor de éxito para LEOCH. La distancia pielcálculo, IMC, tamaño y localización del cálculo son variables que no se relacionan con el éxito de la LEOCH. La eficacia global del litotriptor PiezoLith 3000 plus (Richard Wolf) es de 50\%.

\section{REFERENCIAS}

1. Medina-Escobedo M, Zaidi M, Real-de Leon E, OrozcoRivadeneyra S. [Urolithiasis prevalence and risk factors in Yucatan, Mexico]. Salud Publica Mex 2002;44(6):541-5.

2. Basri Cakiroglu, Erkan Eyyupoglu, Tuncay Tas, Orhun Sinanoglu, Ismet Hazar, M. Bahadir Can Balci, et al. The influence of stone size, skin to stone distance and hydronephrosis on extracorporeal shock wave lithotripsy session and shock wave numbers in ureteral stones. World J Nephrol Urol. 2013;2(2):60-4.

3. Wiesenthal JD, Ghiculete D, Honey RJ, Pace KT. Evaluating the importance of mean stone density and skin-to-stone distance in predicting successful shock wave lithotripsy of renal and ureteric calculi. Urol Res. 2010;38(4):307-13.

4. Vivaldi B, Fernandez MI, Lopez JF, Fuentes F, Urzua C, Krebs $A$, et al. [Single-session extracorporeal shock wave lithotripsy for urinary calculi: factors predicting success after three weeks of follow-up]. Actas Urol Esp. 2011;35(9):529-33. 
5. Pareek G, Hedican SP, Lee FT, Jr., Nakada SY. Shock wave lithotripsy success determined by skin-to-stone distance on computed tomography. Urology. 2005;66(5):941-4.

6. Wang LJ, Wong YC, Chuang CK, Chu SH, Chen CS, See LC, et al. Predictions of outcomes of renal stones after extracorporeal shock wave lithotripsy from stone characteristics determined by unenhanced helical computed tomography: a multivariate analysis. Eur Radiol. 2005;15(11):2238-43.

7. Kanao K, Nakashima J, Nakagawa K, Asakura H, Miyajima $A$, Oya $M$, et al. Preoperative nomograms for predicting stone-free rate after extracorporeal shock wave lithotripsy. J Urol. 2006;176(4 Pt 1):1453-6; discussion 6-7.

8. Yoshida S, Hayashi T, Ikeda J, Yoshinaga A, Ohno R, Ishii N, et al. Role of volume and attenuation value histogram of urinary stone on noncontrast helical computed tomography as predictor of fragility by extracorporeal shock wave lithotripsy. Urology. 2006;68(1):33-7.

9. Perks AE, Schuler TD, Lee J, Ghiculete D, Chung DG, RJ $\mathrm{DAH}$, et al. Stone attenuation and skin-to-stone distance on computed tomography predicts for stone fragmentation by shock wave lithotripsy. Urology. 2008;72(4):765-9.

10. Weld KJ, Montiglio C, Morris MS, Bush AC, Cespedes RD. Shock wave lithotripsy success for renal stones based on patient and stone computed tomography characteristics. Urology. 2007;70(6):1043-6; discussion 6-7.
11. Perks AE, Gotto G, Teichman JM. Shock wave lithotripsy correlates with stone density on preoperative computerized tomography. J Urol. 2007;178(3 Pt 1):912-5.

12. Yazici O, Tuncer M, Sahin C, Demirkol MK, Kafkasli A, Sarica K. Shock wave lithotripsy in ureteral stones: evaluation of patient and stone related predictive factors. International Braz J Urol . 2015;41(4):676-82.

13. Ucer O, Ceylan Y, Ekren F, Ozan E, Muezzinoglu T. Effect of anxiety and pain on success of shockwave lithotripsy (SWL) for treatment of proximal ureteral and renal pelvic stones. Urolithiasis. 2016;44(6):559-64.

14. Wu H, Wang J, Lu J, Wang Y, Niu Z. Treatment of renal stones $>/=20 \mathrm{~mm}$ with extracorporeal shock wave lithotripsy. Urol Int. 2016;96(1):99-105.

15. Kim JK, Ha SB, Jeon CH, Oh JJ, Cho SY, Oh SJ, et al. Clinical nomograms to predict stone-free rates after shock-wave lithotripsy: Development and internal-validation. PloS One. 2016;11(2):e0149333.

16. Bengio RG, Arribillaga L, Epelde J, Orellana S, Garcia-Onto $\mathrm{H}$, Montedoro A, et al. [Predictive score of success adapted to our environment to improve results of extracorporeal lithotripsy]. Arch Esp Urol. 2016;69(7):398-404.

\section{AVISO PARA LOS AUTORES}

Revista Mexicana de Urología tiene una nueva plataforma de gestión para envío de artículos: https://www.revisionporpares.com/index.php/RMUrol ahí podrá inscribirse a la base de datos administrada por el sistema Open Journal System (OJS) que ofrece las siguientes ventajas para los autores:

- Subir sus artículos directamente al sistema.

- Conocer, en cualquier momento, el estado de los artículos enviados, es decir, si ya fueron asignados a un revisor, aceptados con o sin cambios, o rechazados.

- Participar en el proceso editorial corrigiendo y modificando sus artículos hasta su aceptación final. 